\title{
Development of the calibration device using UAV mounted UV-LED light source for the fluorescence detector
}

\author{
Takayuki Tomida ${ }^{1, *}$, Taichi Inadomi $^{1}$, Terutsugu $\mathrm{Seki}^{1}$, Yuichiro Tameda ${ }^{2}$, Yuya $\mathrm{Oku}^{2}$, Kengo Sano ${ }^{1}$, and TA Collabora- \\ tion \\ ${ }^{1}$ Shinshu University, Nagano, Nagano, Japan \\ ${ }^{2}$ Osaka Electro-Communication University, Neyagawa, Osaka, Japan
}

\begin{abstract}
We have developed a standard UV-LED light source mounted on an unmanned aerial vehicle (UAV), so-called the Opt-copter, for the calibration of the fluorescence detectors (FDs) of the Telescope Array experiment. The positioning accuracy of the UAV controlled by GPS is $\sim 10 \mathrm{~cm}$, which enables a precision calibration of the pointing directions of the FD phototubes. We report the hardware details of the device and the status of data analysis.
\end{abstract}

\section{Introduction}

The Telescope Array (TA) experiment located in Utah, USA aims at observing ultra-high-energy cosmic rays (UHECRs) with energies greater than $10^{18} \mathrm{eV}$. TA utilizes two types of detectors, the fluorescent detectors (FDs) in three stations that measure fluorescens photons emitted by air molecules along a cosmic ray shower, and the surface detectors (SDs) that measure shower particles at the ground. The TA detectors are operational since May 2018 [1][2].

This report deals with the calibration systems for the TA FDs. The accuracy of the optical systems and phototube gains are key in the determination of arrival directions and energies of cosmic rays. One of the common methods to calibrate pointing directions of optical sensors is to use light sources whose positions are previously known with good accuracies, like stars. We developed a FD calibration system with a high-luminosity UV LED mounted on an unmanned aerial vehicle (UAV) controlled by GPS with the accuracy of $\sim 10 \mathrm{~cm}$. Its position stability and portability enables us to calibrate the optical system of our FDs with good pointing accuracies by a single standard light source for all the FDs at the three sites separated by 35 $\mathrm{km}$ each other. The conceptual image of this device, Optcopter, is shown in Fig. 1.

\section{The Opt-copter}

The Opt-copter is composed of an unmanned aerial vehicle (UAV), a UV-LED as the light source, and several GPS modules (Fig. 2). The position accurary of the Opt-copter enables the calibration of FD phototubes with the accuracy of 0.1 degrees or even better.

*e-mail: tomida@cs.shinshu-u.ac.jp

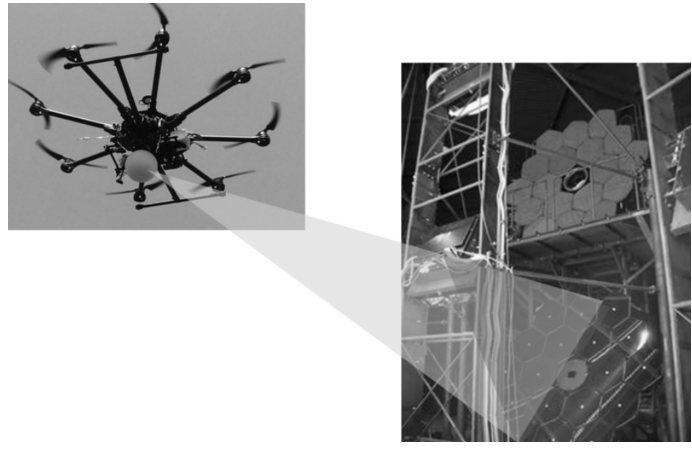

Figure 1. The conceptual image of measurement by Opt-copter.

\subsection{UAV}

We use a high-stability 8-rotor helicopter (DJI S1000+) to load the light source. The size of the UAV $400 \times 400 \times 500$ $\mathrm{mm}(\mathrm{WxDxH})$ when the arms collapsed, and its portability with automatic leg and centrifugal propeller folding mechanism helps works in wilderness at night. Both operations by manual control and by a programmed flight path are possible. A high-power and high-efficiency heat radiation motor and the all-carbon body frame of the vehicle realize a flight of $12 \sim 15$, with a $16000 \mathrm{mAh}$ lithium polymer battery. This $S 1000+$ is also designed to load a camera for aerial photography, and we use this room $\left(\sim 30 \mathrm{~cm}^{3}\right)$ to mount the light source and electronic devices. The maximum load weight is $7 \mathrm{~kg}$.

\subsection{The flight controller}

The flight controller (DJI A3) consists of a GPS and an attitude sensor to measure acceleration and atmospheric pressure. The positioning accuracy of $S 1000+$ with $A 3$ is about $3 \mathrm{~m}$. 


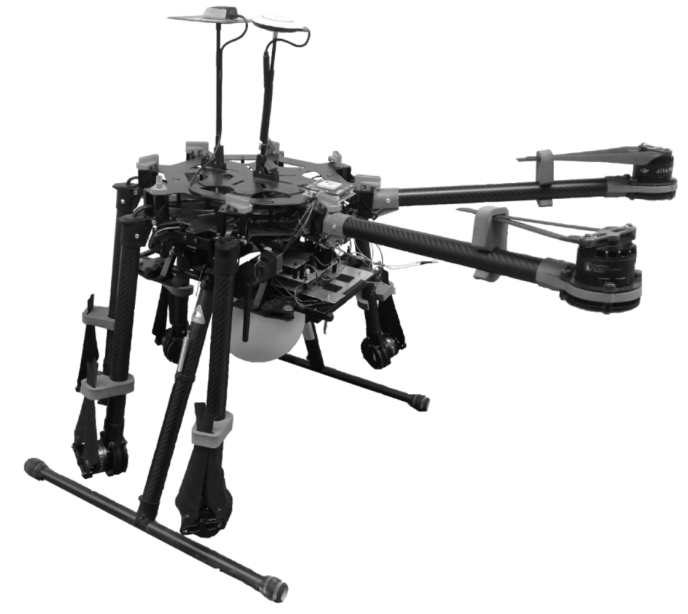

Figure 2. Opt-copter has 8 arms and all of them are able to be folded

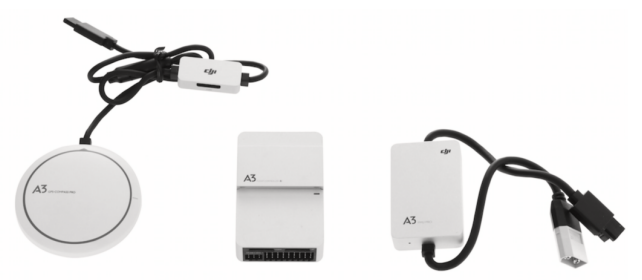

Figure 3. Appearance of $\mathrm{A} 3$ as a UAV control device using GPS.

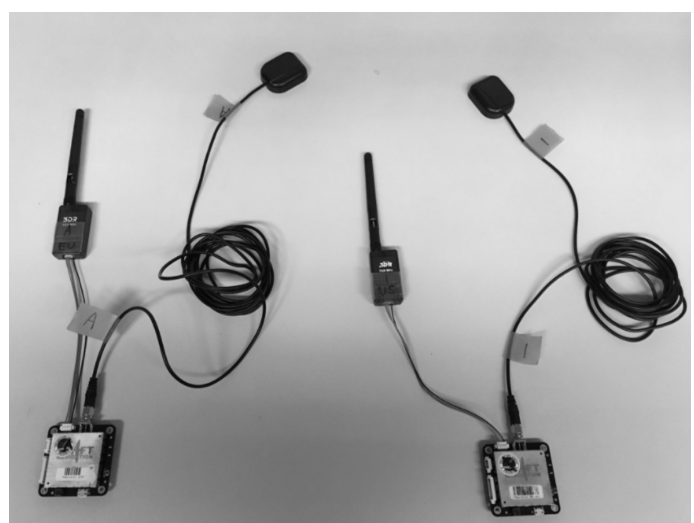

Figure 4. piksi is composed of two modules and record the relative proposition of them

\subsection{RTK-GPS}

The opt-copter is designed to hover $300 \mathrm{~m}$ ahead of the FDs in calibration flights. The positioning accuracy of 3 $\mathrm{m}$ corresponds to a pointing accuracy of $0.6^{\circ}$ at the distance of $300 \mathrm{~m}$ (c.f. the field of view of each phototube is $1^{\circ}$ ). For a phototube pointing calibration with the accuracy of $0.1^{\circ}$, a positioning accurary of $0.5 \mathrm{~m}$ is needed, and the Real Time Kinetic GPS (RTK-GPS) system (Swift Navigation, Piksi) enables this (Fig. 5). RTK-GPS consists

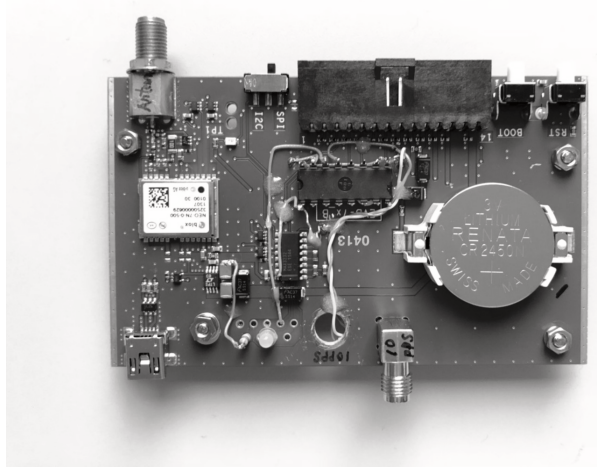

Figure 5. Appearance of gps-based pulse generator (ublox, EVK-M8-TCXO)

of two GPS modules, and records the relative position of the GPS antennas using the phase difference of the signals emitted from the GPS satellites. The position accuracy of RTK-GPS is typically $10 \mathrm{~cm}$ after GPS calibration more than 1.5 hours. We evaluated the positioning accuracy on the ground. The movement distance was measured $2 \mathrm{~m}$ and 10 moves, and RTK-GPS was calibrated for each of the tests and tried 10 times. The distribution of the measured relative distances between the two modules is shown in Fig. 6, which exibits that the horizontal and vertical accuracy of RTK-GPS is better than $10 \mathrm{~cm}$. By loading one GPS module of RTK-GPS on the UAV and placing the other at a reference point on the ground where the position is previously measured in good accuracy, it is possible to know actual positions of the UAV and the direction seen from the FDs.

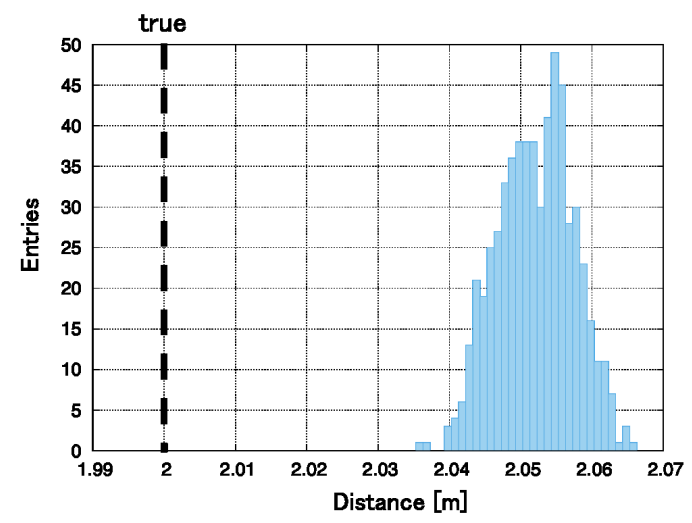

Figure 6. Ranging accuracy of RTK-GPS on the ground.

\subsection{The triggering system}

The TA FD consists of a light collecting mirror of $3.3 \mathrm{~m}$ diameter and a $16 \times 16$ cluster of phototubes (the camera). The FD is designed to trigger the data acquisition system when photons are detected with more than 5 adjoining phototubes within 25.6 mus to detect cosmic ray showers, or by an external signal to the FD. Since the size 


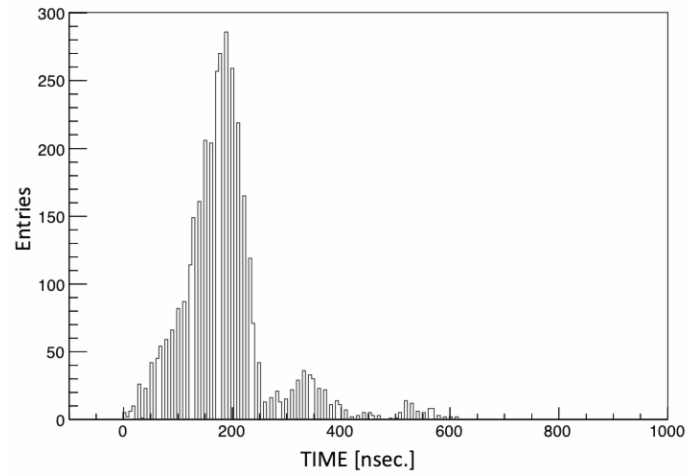

Figure 7. Time difference between pulse generator for light source and RTK-GPS measurment for ranging.

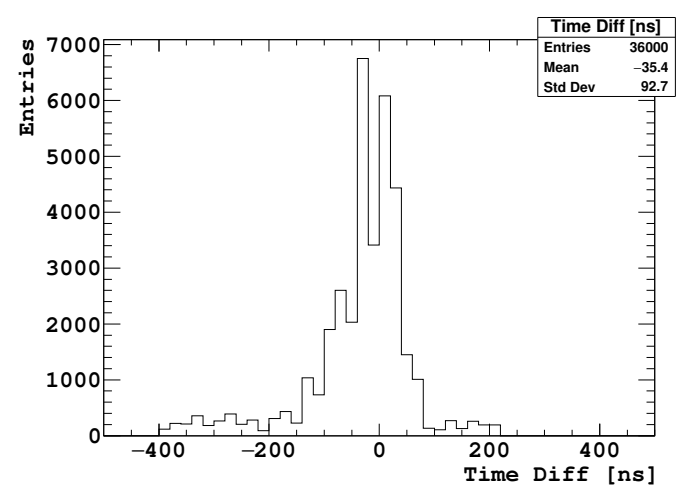

Figure 8. Time difference between pulser for FD trigger and pulser for light source.

of the Opt-copter light source image on the FD camera is as small as the size of a phototube, which was confirmed by our preparatory measurement hovering the UAV at the center of the field of view of an FD, the self-triggering of FD does not work for Opt-copter signals. Therefore we need a trigger generator for this to send trigger pulses both to FD and the light source in order that a measurement of the light source position, a UV-LED flash, and the FD data acquisition are made at the same time. The position measurements by the RTK-GPS as at the frequency of $10 \mathrm{~Hz}$, and we use two GPS-based pulse generator of $10 \mathrm{~Hz}$, one on the Opt-copter for LED flashes, and the other is to trigger the FD data acquisition. This frequency is well below the maximum trigger rate of the TA FD, $30 \mathrm{~Hz}$. All the three GPS modules are presumably synchronized by the GPS-PPS signal every second. We compared the signal timing differences using an external high precision pulse generator that is also synchronized with GPS-PPS, and the GPS pulse for the Opt-copter as shown in Fig. 7. The time differences between the two GPS-based pulse generator, for the Opt-copter and for the FD trigger, is presented in Fig. 8. This shows that the synchronization of the GPSbased pulse generator is as good as $0.1 \mu \mathrm{s}$, which is much smaller than the width of the UV-LED flash.

\subsection{UV-LED light source}

The optical system of the TA FD is optimzed for photons of wavelengths between 300 and $400 \mathrm{~nm}$, for fluorescence light from nitrogen and oxgen molecules. We use 12 UV-LEDs (Roithner Lasertechnik, H2A1-H375-E) at wavelength of $375 \mathrm{~nm}$. The emission pattern of each LED is highly anisotropic, and we use a spherical light diffuser to minimize the UAV attitude dependence of light intensity seen from the FDs. The diffuse is dodecahedron in shape by 3D printer made of acrylic resin, and the LEDs are attached on every side of the diffuser (Fig. 9).

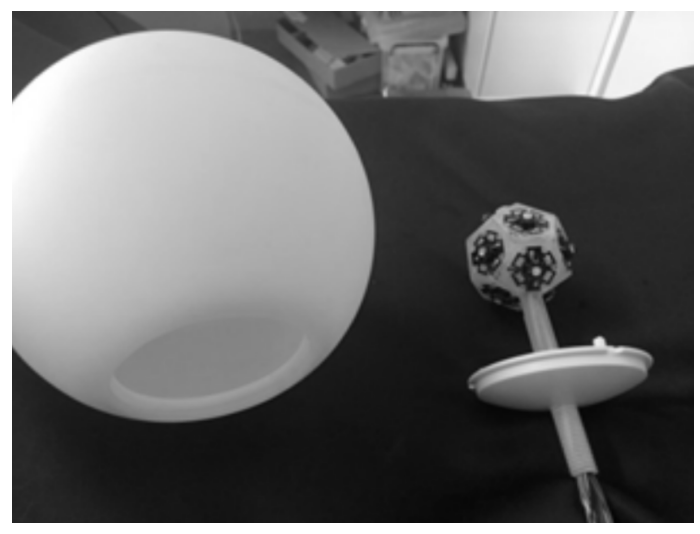

Figure 9. The light source mounted on the Opt-copter is consisted of 12 UV-LEDs attached on dodecahedron and a spherical diffuser

\section{Operation and data}

The position of the launching point of the Opt-copter ahead of each FD station is measured with good accuracy in advance. The light source is designed to be seen from the FD at the distance of $300 \mathrm{~m}$, and the vehicle flies around in the field of view of the camera with a positioning accuracy of $10 \mathrm{~cm}$, which corresponds to a directional accuracy of $0.02^{\circ}$. Fig. 10 shows a bird's eye view of an Opt-copter flight.

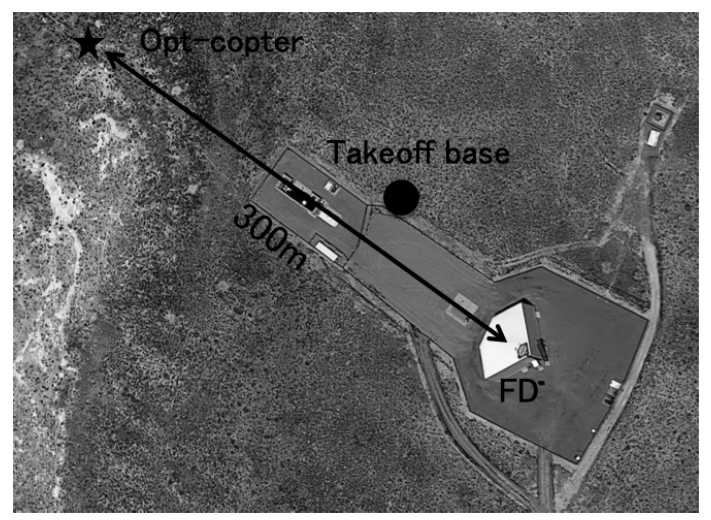

Figure 10. A bird's-eye view of the flight

The position information obtained by the Opt-copter is obtained from the FD and the RTK-GPS. The center of 


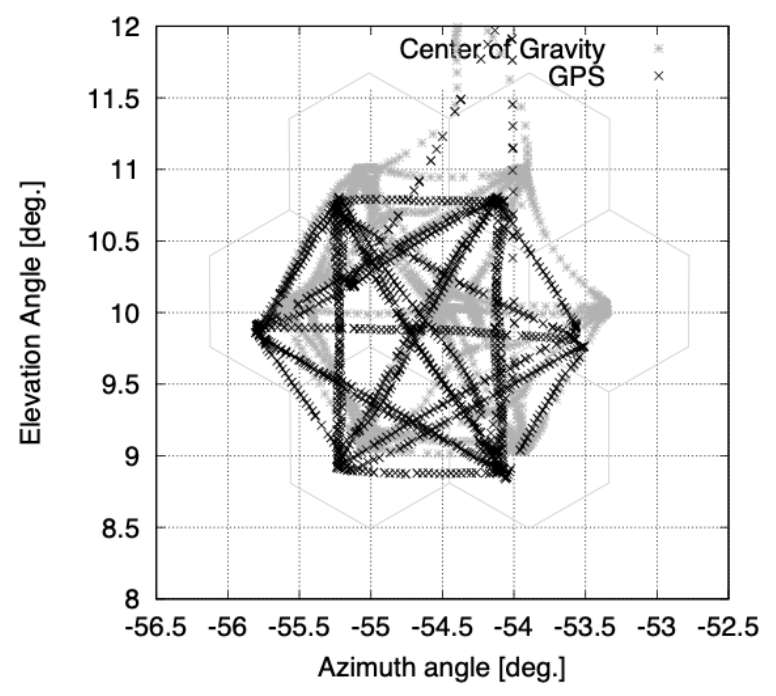

Figure 11. Measurement position by RTK-GPS on FD viewing field (Gray) and center of gravity of detected light by FD (Light gray).

gravity of the amount of received light indicates the position of the light source, and the RTK-GPS measures the relative position of the GPS antenna on the Opt-copter and the GPS antenna on the ground reference station. To compare the two, we project information by the RTK-GPS on the FD view (See Fig. 11.). The trajectory of the detected center of gravity appears to be biased to the center of each PMT, which is different from the position of the projected image by the RTK-GPS measurement. If the image of the light source is sufficiently smaller than the size of one pixel of the FDs (PMT), the center of gravity is biased towards the center of the PMT, which contains the main part of the image of the light source. By measuring the centerof-gravity shift when the measurement by the RTK-GPS is taken as the true position, it is expected that the optical characteristics of the FD such as the difference of the focused spot can be obtained. We evaluate the correlation of the opening angles between the RTK-GPS and the center of gravity from the view center of the FD. Figure 12 shows data of two FDs as examples. This difference is consistent with the construction records. In other words, it is suggested from the relationship between the RTK-GPS and the light receiving gravity center of the FD that it has sensitivity to the size of the focused spot in the vicinity of the visual field center of the FD. The plot shifts horizontally from the solid line in Fig. 12 if the field of view of our assumed FD and the actual field of view deviate.

\section{Discussion}

The Opt-copter showed the measurement performance of geometrical optics parameters for the current TA-FD. Future analysis will provide fine optical characteristics of FD. As a result, more advanced calibration can be expected, reducing energy shower energy and systematic er-
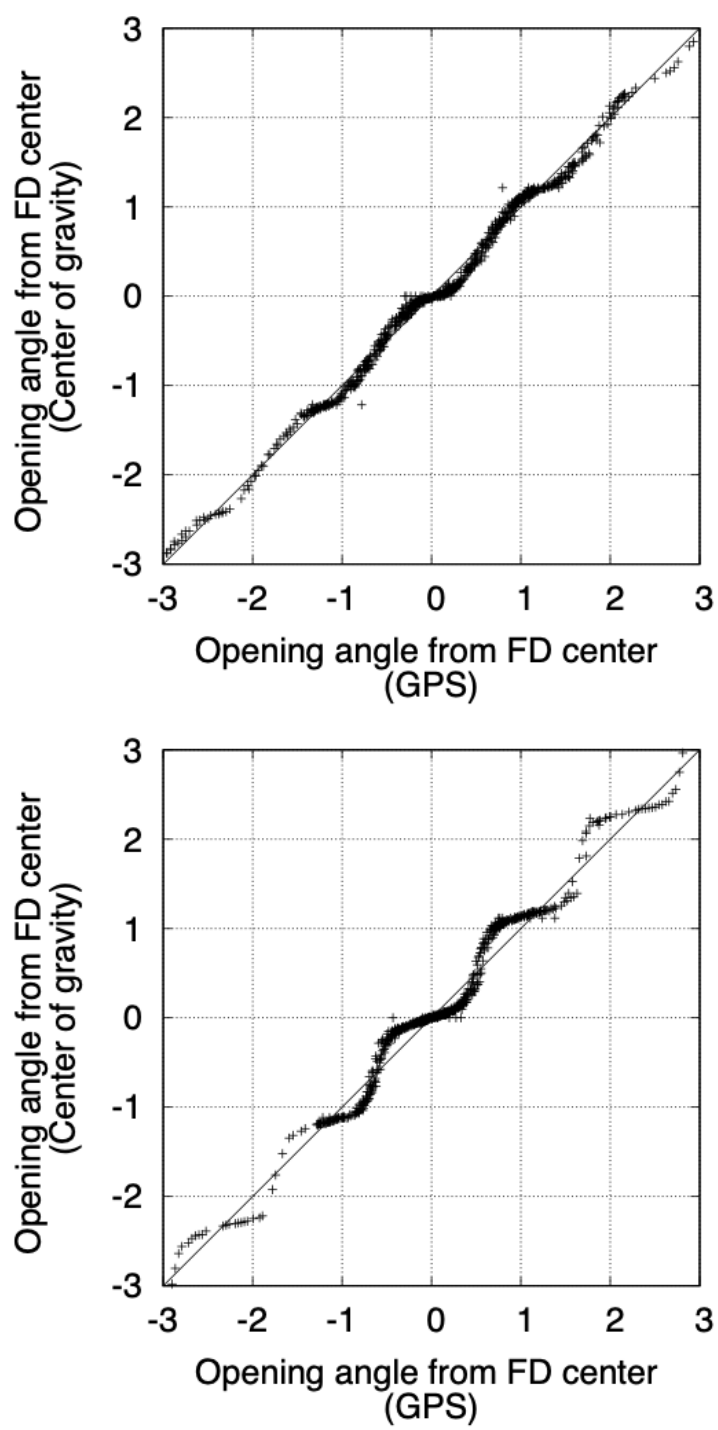

Figure 12. Correlation of opening angle from the center of field of view of RTK-GPS and FD of detection center of gravity in two FDs.

ror of Xmax is expected.

\section{Acknowledgements}

The Telescope Array experiment is supported by the Japan Society for the Promotion of Science(JSPS) through Grants-inAid for Priority Area 431, for Specially Promoted Research JP21000002, for Scientific Research (S) JP19104006, for Specially Promoted Research JP15H05693, for Scientific Research (S) JP15H05741 and for Young Scientists (A) JP17K14280 and for Young Scientists (B) JPH26707011; by the joint research program of the Institute for Cosmic Ray Research (ICRR), The University of Tokyo; by the U.S. National Science Foundation awards PHY-0601915, PHY-1404495, PHY1404502, and PHY-1607727; by the National Research Foundation of Korea (2016R1A2B4014967, 2016R1A5A1013277, 
2017K1A4A3015188, 2017R1A2A1A05071429) ; by the Russian Academy of Sciences, RFBR grant 16-02-00962a (INR), IISN project No. 4.4502.13, and Belgian Science Policy under IUAP VII/37 (ULB). The foundations of Dr. Ezekiel R. and Edna Wattis Dumke, Willard L. Eccles, and George S. and Dolores Doré Eccles all helped with generous donations. The State of Utah supported the project through its Economic Development Board, and the University of Utah through the Office of the Vice President for Research. The experimental site became available through the cooperation of the Utah School and Institutional Trust Lands Administration (SITLA), U.S. Bureau of Land Management (BLM), and the U.S. Air Force. We appreciate the assistance of the State of Utah and Fillmore offices of the BLM in crafting the Plan of Development for the site. Patrick Shea assisted the collaboration with valuable advice on a vari- ety of topics. The people and the officials of Millard County, Utah have been a source of steadfast and warm support for our work which we greatly appreciate. We are indebted to the Millard County Road Department for their efforts to maintain and clear the roads which get us to our sites. We gratefully acknowledge the contribution from the technical staffs of our home institutions. An allocation of computer time from the Center for High Performance Computing at the University of Utah is gratefully acknowledged.

\section{References}

[1] H.Tokuno et.al., NIM A, 676, 54-65 (2012)

[2] T.Abu-Zayyad, et.al., NIM A, 689, $87-97$ (2012) 